\title{
Desarrollo de filtro pasa banda larga con ceros de transmisión para sistemas de comunicación Ultra- Wideband
}

\author{
Sara María Yepes Zuluaga \\ MsC en Telecomunicaciones, \\ Universidad de Brasilia \\ Docente Tiempo Completo, Investigador Grupo GIT, \\ Instituto Tecnológico Metropolitano ITM \\ Medellín, Colombia \\ sarayepes@itm.edu.co
}

\begin{abstract}
Resumen- Este artículo presenta el diseño e implementación de un filtro pasa banda con dos secciones de stubs en circuito abierto para aplicaciones Ultra wideband - UWB. EI filtro que se utilizó inicialmente se basa en un dispositivo con sección de stubs en corto circuito, y con una aplicación en algoritmos genéticos (AG) se obtuvieron valores optimizados de impedancias de las secciones, para transformarse en dos secciones de stubs en circuito abierto, que facilita su fabricación. El diseño del filtro de grado $\mathbf{4 0}$ se implementó en líneas microcintas y las respuestas medidas de los parámetros de transmisión y reflexión mostraron un comportamiento próximo a las respuestas simuladas, es un filtro de tamaño reducido, con un gran ancho de banda.

Palabras clave-: Filtro pasa banda, Ultra Wideband (UWB), Microcinta, stubs.

Abstract-This paper presents the band pass filter using two stub sections in an open circuit configuration, for UItra Wideband technology applications. Filter is based on a device with a short circuit stub section. Using Genetic Algorithms (AG) theory values were obtained for optimal impedance stub sections, which were used to transform the initial filter in a two section open circuit stub device. Third and fourth degree filters were designed and implemented on a microstrip line technique and their response measurements revealed a very close behavior to the simulated responses.
\end{abstract}

Keywords- Band pass filter, Ultra Wideband, Microstrip, stub.

\section{INTRODUCCIÓN}

UWB es una tecnología inalámbrica utilizada para redes de área personal de corto alcance (Wireless Personal Area Networks-WPAN) que ofrece una combinación de buen funcionamiento y de uso fácil. La Comisión Federal de Comunicaciones [1], emitió un mandato para que la transmisión de radio en UWB pudiese operar en la banda de 3.1 hasta $10,6 \mathrm{GHz}$, con una potencia de transmisión de $-41 \mathrm{dBm} / \mathrm{MHz}$, además, determinó que la ocupación de la banda de UWB tendría que ser mayor a $500 \mathrm{MHz}$, con un ancho de banda fraccional mayor al $20 \%$, parámetros que fueron considerados en el diseño del filtro.

Diferentes estructuras de filtros han sido desarrollados para aplicaciones en los sistemas ultra wideband (UWB). Se presentan varios filtros microcinta pasa-banda para ser aplicados en esta tecnología. En [2] es utilizado el filtro pasa-alto óptimo distribuido, de 11. orden con un tamaño reducido y de alta selectividad, en su implementación se presenta una alta dificultad al fabricarse en microcinta, motivo por el cual se convertir las secciones de stubs del filtro inicial de este trabajo en stubs de circuito abierto. Otra estructura que han presentado es un diseño de filtro pasa banda wideband a $3,4 \mathrm{GHz}$, usando dos secciones de stubs en circuito abiertos en configuración shunt con líneas microcinta [3], de fácil diseño y construcción, pero no cubre el ancho de banda total requerido por UWB. También han diseñado un novedoso filtro pasa banda ultra wideband en tecnología microcinta con stubs cortocircuitados de $\lambda / 4$ para mejorar el rendimiento y reducir el tamaño del filtro UWB basado en las teorías tradicionales [4], en su diseño se genera los ceros de transmisión para atenuar las bandas de rechazo, pero posee una alta complejidad en su fabricación. En el artículo [5] es implementado un filtro pasa-banda con el resonador multi-modo (MMR), también llamado resonador stepped-impedance (SIR), con dos líneas de cuarto de longitud de onda acopladas en paralelo en la entrada y en la salida de las puertas. Teniendo como resultado un filtro con un muy buen ancho de banda fraccional de $113 \%$ y una frecuencia central de $6,85 \mathrm{GHz}$, con 
muy baja selectividad. En [6] se presenta un filtro en microcinta con aplicación en UWB combinando el filtro pasa-bajo con el filtro pasa-alto y en [7] se presenta una mezcla de filtros pasa-banda y rechaza banda broadband en cascada, que no alcanzan a obtener el ancho de banda requerido por la FCC para UWB. Dentro de los métodos utilizados para la optimización de los filtros mencionados con anterioridad están: por ejemplo en [8] los valores de las impedancias características de las líneas de transmisión son optimizadas en un filtro mediante la función de transferencia en el dominio $Z$, aunque uno de los métodos más comunes para la optimización es el proceso auto-regresivo (AR) y errores de mínimos cuadrados (LSM) que es utilizado en [9], en este caso, fue empelado el algoritmo genético de optimización desarrollado por un grupo de investigación de la universidad de Brasilia. El algoritmo fue adaptado para la optimización del diseño de filtros en microcintas para frecuencias de microondas. Las respuestas simuladas y medidas para los parámetros $\mathrm{S}_{11}$ y $\mathrm{S}_{21}$ fueron satisfactorias, comprobada la efectividad del algoritmo genético al ser empleado en la optimización de los filtros.

El diseño del filtro inicial se basó en el filtro pasa-alto distribuido construido por una cascada de stubs en corto circuito y de líneas unitarias. Inicialmente se realizó la optimización de los valores de las impedancias características de cada sección de la línea de transmisión del filtro con una herramienta de optimización como lo es el algoritmo genético previamente desarrollado y adaptado en este tipo de filtro. Para mejorar la respuesta del filtro es necesario obtener una mayor atenuación en la banda de rechazo del filtro y producir ceros de transmisión en las frecuencias bajas y altas de dicha banda, los stubs en corto circuito fueron reemplazados por dos secciones de stubs en circuito abierto, haciendo más fácil su fabricación. Las simulaciones del circuito abierto se realizaron en el software Microwave Studio [10I, los parámetros obtenidos de estas simulaciones fueron $\mathrm{S}_{11}$ y $\mathrm{S}_{21}$, así como el diseño del circuitos para su fabricación. Posterior a la construcción del circuito fueron verificados los parámetros a partir de las mediciones, las cuales fueron comparadas con los datos simulados, se evidencia una diferencia menor del 30\%.

Dentro de las características a resaltar de dispositivo desarrollado están: la simpleza, lo compacto de la estructura y la facilidad de fabricación.

\section{METODOLOGÍA}

Para que la implementación del filtro pasa banda utilizado en la aplicación de los sistemas UWB sea clara, es imprescindible analizar el comportamiento del filtro pasa alto distribuido presentado en la Fig. 1.

FIG. 1. CONFIGURACIÓN DE UN FILTRO PASA ALTO DISTRIBUIDO DE $4 .^{\circ}$ GRADO, CON DOS STUBS EN CORTO CIRCUITO.

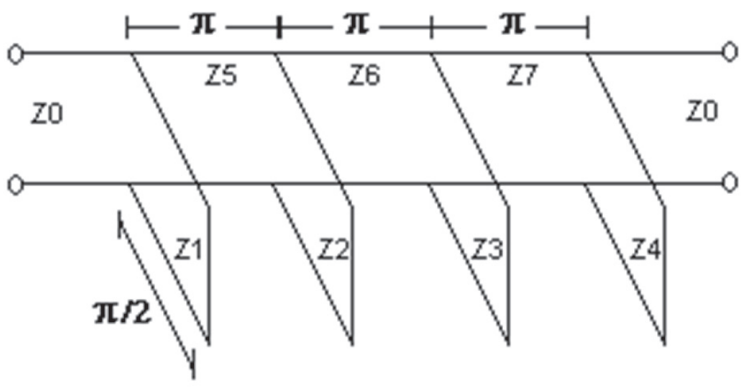

Las características del filtrado en la red de la Fig.1, pueden ser descritas por la función de transferencia expresada por [11]:

$$
\mid S_{21}(\theta)^{2}=\frac{1}{1+\varepsilon^{2} F_{N}{ }^{2}(\theta)}
$$

Donde, $\mathrm{N}$ es el grado del filtro, o sea, el número de stubs en corto circuito, $\varepsilon$ es la constante de rizado en la banda pasante, $\theta$ es la longitud de onda eléctrica que es proporcional a la frecuencia variable y $F_{N}(\theta)$ es la función del filtrado obtenido por:

$$
F_{N}(\theta)=\frac{\left(1+\sqrt{1-x_{c}^{2}}\right) T_{2 n-1}\left(\frac{x}{x_{c}}\right)-\left(1-\sqrt{1-x_{c}^{2}}\right) T_{2 n-3}\left(\frac{x}{x_{c}}\right)}{2 \cos \left(\frac{\pi}{2}-\theta\right)}
$$

Donde

$$
x=\operatorname{sen}\left(\frac{\pi}{2}-\theta\right), x_{c}=\operatorname{sen}\left(\frac{\pi}{2}-\theta_{c}\right)
$$

$\theta_{c}$ es la longitud de onda eléctrica en la frecuencia de corte, $T_{n}=\cos \left(n \cos ^{-1} x\right)$ es la función de Chebyshev de primer tipo de grado $\mathrm{n}$.

Teóricamente, este tipo de filtro pasa alto puede ser un pasa banda primario extremamente grande. Así $\theta_{c}$ puede asumir valores muy pequeños, para que esto ocurra es necesario que los stubs en corto circuito tengan niveles de impedancia altos. 
Este tipo de filtro fue optimizado, para ello existen diferentes métodos de optimización de sistemas, el método escogido depende de las características del problema a ser optimizado. Dentro de los diversos métodos se encuentra los algoritmos genéticos (AGs), caracterizados como métodos estocásticos. El objetivo del algoritmo genético es encontrar el valor óptimo de una determinada función sobre un espacio de búsqueda.

\section{A. Definición de la función objetivo en AG}

Las características físicas de la estructura del filtro pasa banda se consideran como condiciones iníciales del AG, y en la herramienta de cálculo son clasificadas como variables las impedancias de las líneas unitarias y las impedancias de los stubs en corto circuito, estableciéndose un rango de valores entre 20 y 80 para realizar la variación de cada uno de los valores.

Las dimensiones de las líneas unitarias son definidas con una longitud de onda eléctrica de $\pi$ y cada stub en corto circuito tiene una longitud de onda eléctrica de $\pi / 2$, (ver Fig. 1). El número de generaciones indica el número de interacciones en que la rutina de optimización es interrumpida. Durante el proceso de evolución, en cada generación, los valores evaluados de los individuos son diferentes hasta que finalmente convergen alrededor de una solución óptima. Se emplearon 50 generaciones. El número de individuos y el tamaño de la población es el parámetro que determina el número posible de soluciones para el problema. Se consideraron 50 individuos para cada generación.

En este caso, la función objetivo es la respuesta deseada del parámetro de transmisión y del parámetro de reflexión del filtro pasa alto distribuido con dos líneas unitarias de $\lambda / 2$ de 4 stubs de $\lambda / 4$ en corto circuito. En la herramienta se define la función objetivo que establece los parámetros $\mathrm{S}_{11}$ y $\mathrm{S}_{21}$ deseados, (ver Fig. 2). Se obtiene los valores de las impedancias optimizadas de todas las secciones de líneas de transmisión del filtro de la Fig. 1, que corresponden a las mejores respuestas de la función de transferencia $S_{11}$ y $S_{21}$.
La máscara definida como la función objetivo del algoritmo genético tiene las siguientes características: la banda pasante está delimitada entre las frecuencias $4,1 \mathrm{GHz}$ hasta 8,65 $\mathrm{GHz}$, con un nivel de rizado de $0,5 \mathrm{~dB}$, para la banda de rechazo de 2 a $4 \mathrm{GHz}$ y de 8,65 hasta $12 \mathrm{GHz}$ el nivel de pérdida está entre -30 a -15 dB. En la Fig. 2, se presenta la máscara del parámetro $\mathrm{S}_{11}$ para la banda de $4,1 \mathrm{GHz}$ hasta $8,65 \mathrm{GHz}$, el nivel de pérdida es de $-30 \mathrm{~dB}$.

Con los valores de impedancias entregados por la herramienta de optimización y con los valores de frecuencia escogidos en la banda de rechazo, es posible aplicar la teoría para transformar el filtro con stubs en corto circuito en dos secciones de stubs en circuito abierto, se genera ceros de transmisión en la banda de rechazo.

FIG.2. MÁSCARA DEL PARÁMETRO $\mathrm{S}_{21}, \mathrm{~S}_{11}$ Y MEJOR RESPUESTA OPTIMIZADA PARA EL FILTRO DE $4 .^{\circ}$ GRADO.
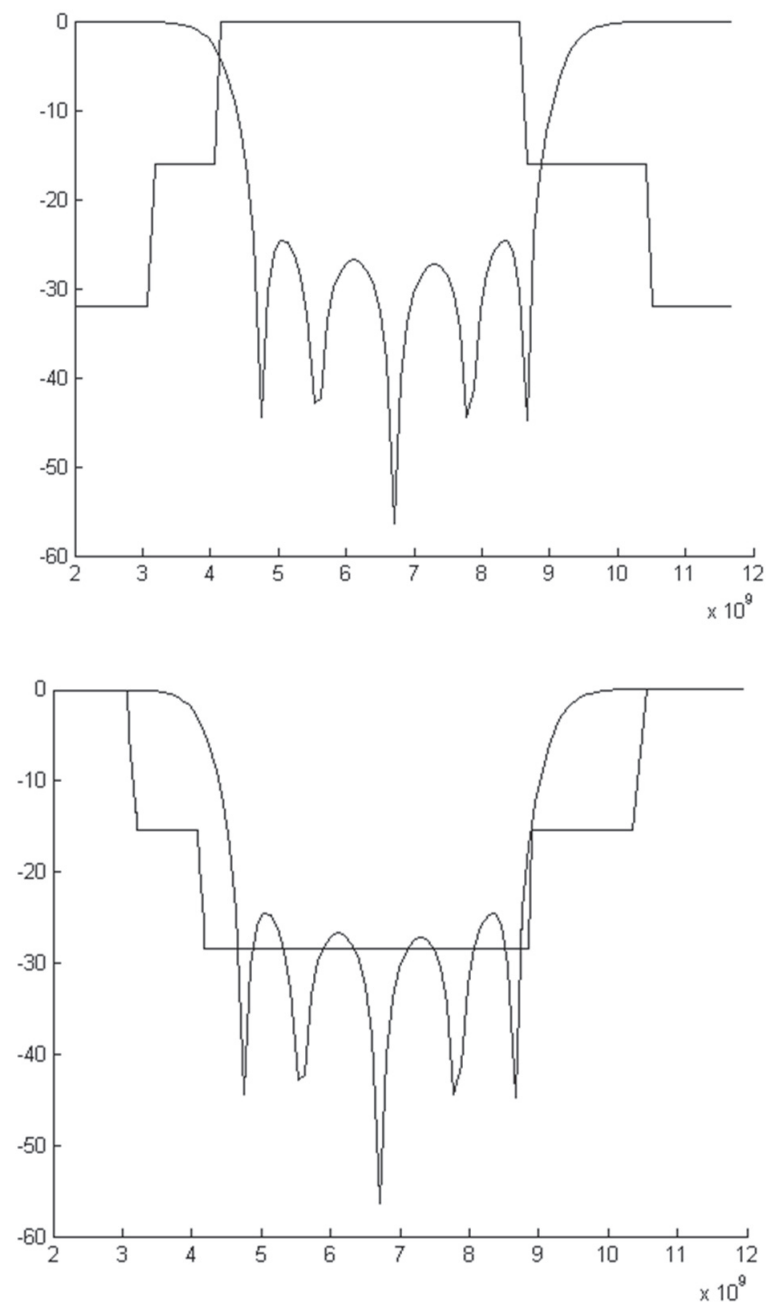
TABLA I. CONDICIONES EMPLEADAS EN LA SIMULACIÓN.

\begin{tabular}{|c|c|}
\hline Tipo de Codificación & Real \\
\hline Operador de cruce & SBX (simulated binary \\
crossover)
\end{tabular}

En la Tabla I están resumidas las condiciones que fueron empleadas para el proceso de optimización, considerada la función objetivo descrita anteriormente.

La herramienta de cálculo fue implementada en una máquina con un procesador Pentium 4 de $3 \mathrm{GHz}$ y memoria de $1 \mathrm{GHz}$. La simulación duró alrededor de 4 horas y mostró como resultado los mejores individuos de la optimización. Después de esta operación, fue necesario estudiar la población final, para analizar resultados individuales, ya que algunas de las respuestas presentadas no estaban dentro de los objetivos del estudio.

En la Fig. 3, se presentan los resultados de los parámetros $\mathrm{S}_{21}$ y $\mathrm{S}_{11}$ de los individuos de una de las generaciones obtenidas mediante el proceso de optimización.

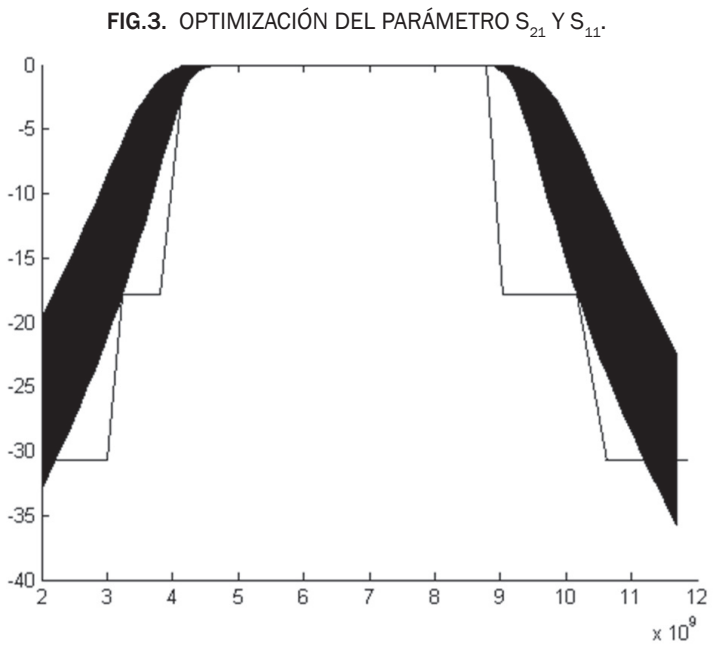

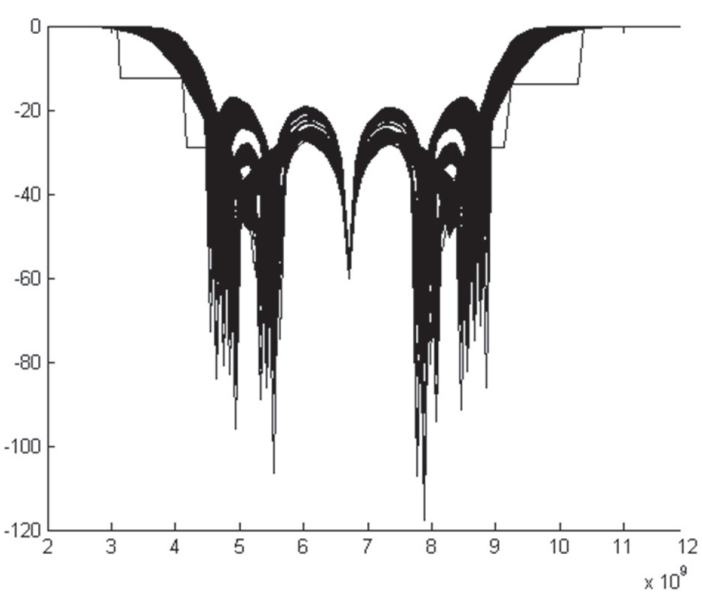

En la Tabla II son presentados los valores de las impedancias que tiene el grupo de las mejores respuestas en el proceso de optimización. Los individuos con mejor desempeño están resaltados en la Tabla II. Estos resultados serán utilizados para el desarrollo de la generación de ceros de transmisión en los filtros con dos secciones de stubs en circuito abierto.

TABLA II. VALORES DE IMPEDANCIAS CON MEJORES RESPUESTAS PARA EL FILTRO DE 4 STUBS.

\begin{tabular}{|c|c|c|}
\hline$Z_{1}=Z_{3}$ & $Z_{2}$ & $Z_{4}=Z_{5}$ \\
\hline $53,71 \Omega$ & $28,52 \Omega$ & $69,02 \Omega$ \\
\hline
\end{tabular}

\begin{tabular}{|c|c|c|c|}
\hline $\mathrm{Z}_{1}=\mathrm{Z}_{4}$ & $\mathrm{Z}_{5}=\mathrm{Z}_{7}$ & $\mathrm{Z}_{2}=\mathrm{Z}_{3}$ & $\mathrm{Z}_{6}$ \\
\hline $44,01 \Omega$ & $76,27 \Omega$ & $22,85 \Omega$ & $79,53 \Omega$ \\
\hline
\end{tabular}

\section{B. Obtención de ceros de transmisión}

Para mejorar la respuesta del filtro y darle una alta selectividad es necesario obtener una atenuación mayor en la banda de rechazo del filtro, es necesario generar ceros de transmisión en las frecuencias determinadas. Para eso, se implementó el uso de dos secciones de stubs en circuito abierto.

El stub en corto circuito produce un par de ceros de transmisión en $\mathrm{f}=0$ y $\mathrm{f}=2 \mathrm{f}_{0}$, donde $\mathrm{f}$ es la frecuencia central del filtro. Para obtener ceros de transmisión en otra frecuencia deseada, se sustituye los stubs en corto circuito con dos secciones de stubs en circuito abierto, como se muestra en la Fig. 4.

Las impedancias equivalentes $Z_{\text {in } 1}$ y $Z_{\text {in } 2}$ del stub en corto circuito y de las dos secciones de 
stubs en circuito abierto. Son expresadas por las ecuaciones (4) y (5) respectivamente [4].

$$
Z_{\text {in } 1}=j Z_{1} \tan \theta
$$

FIG.4. REEMPLAZO DEL STUB EN CORTO CIRCUITO POR LA DOS SECCIONES DE STUB EN ABIERTO.
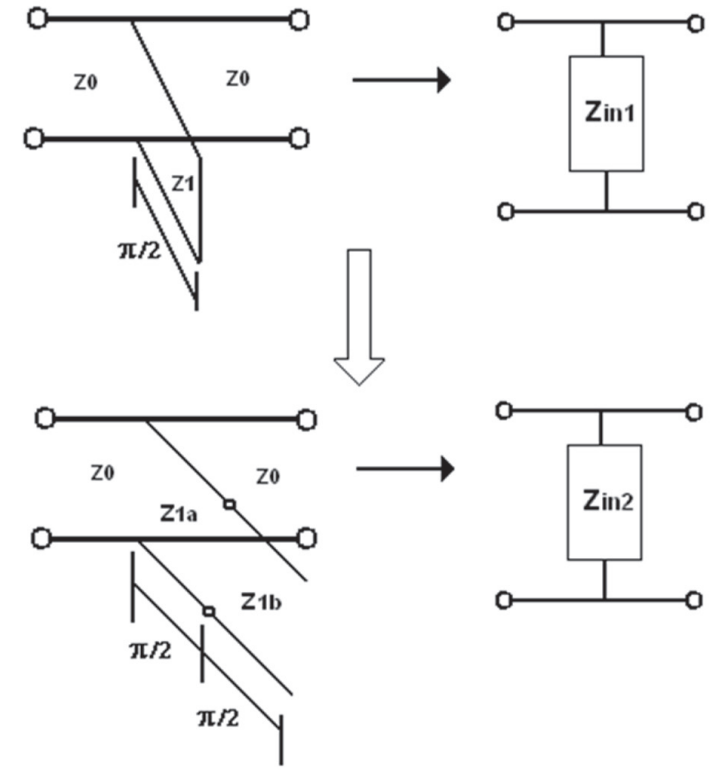<smiles>C1CCCCC1</smiles>

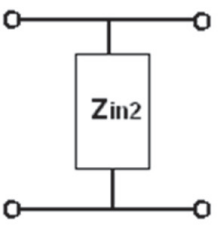

$Z_{i n 2}=j \frac{Z_{1 a}^{2}}{Z_{1 a}+Z_{1 b}} \tan \theta-j \frac{Z_{1 a} Z_{1 b}}{Z_{1 a}+Z_{1 b}} \cot \theta$

Igualándose $Z_{\text {in } 1}$ y $Z_{\text {in2 }}$ en $\mathrm{f}=\mathrm{f}_{0}$ se obtiene:

$$
\frac{Z_{1 a}{ }^{2}}{Z_{1 a}+Z_{1 b}}=Z_{1}
$$

Cada stub en circuito abierto con dos secciones puede producir un par de ceros de transmisión, uno en la frecuencia más baja de la banda de rechazo y el otro en la frecuencia más alta.

En este trabajo se desea obtener un par de ceros en las frecuencias $\theta=\theta 1$ y $\theta=\pi-\theta 1$. Para eso las impedancias $Z_{1 \mathrm{a}}$ y $Z_{1 \mathrm{~b}}$ de la línea de transmisión de las dos secciones del stub en circuito abierto deben satisfacer la siguiente relación:

$$
Z_{1 b}=Z_{1 a} \tan ^{2} \theta_{1}
$$

Sustituyendo (7) en (6), se obtiene:

$$
Z_{1 a}=\left(1+\tan ^{2} \theta_{1}\right) Z_{1}
$$

En la Fig. 5, se presenta la configuración para un filtro de 4 polos con dos secciones de stub en circuito abierto.
En este trabajo se obtuvo tres ceros de transmisión en la frecuencia altas y bajas de la banda de rechazo respectivamente.

FIG.5. CONFIGURACIÓN DEL FILTRO CON STUB DE DOS SECCIONES EN CIRCUITO ABIERTO.

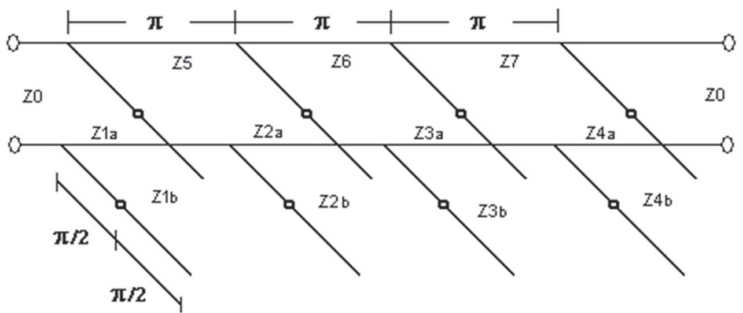

El filtro pasa banda de la Fig. 6, con 4 stubs respectivamente tienen una frecuencia central de $6,85 \mathrm{GHz}$, con una banda de operación de 4,1 $\mathrm{GHz}$, hasta $8,65 \mathrm{GHz}$, y el rizado de $0,5 \mathrm{~dB}$. Los valores optimizados de las impedancias características de los stubs en corto circuito y de las líneas unitarias del filtro de $4 .^{\circ}$ de la Fig.1, son:

\begin{tabular}{|c|c|c|c|}
\hline$Z_{1}=Z_{4}$ & $Z_{5}=Z_{7}$ & $Z_{2}=Z_{3}$ & $Z_{6}$ \\
\hline $44,01 \Omega$ & $76,27 \Omega$ & $22,85 \Omega$ & $79,53 \Omega$ \\
\hline
\end{tabular}

Con los ceros de transmisión definidos en la frecuencias 2, 2,5, 3,1 GHz en la banda baja de rechazo, y 10,6, 11,2, 11,7 GHz en la banda alta de rechazo se calculó las impedancias de las dos secciones de stubs en circuito abierto del filtro de orden $4^{\circ}$. Los valores están dados en $(\Omega)$ :

\begin{tabular}{|c|c|c|c|}
\hline$Z_{1 \mathrm{a}}$ & $\mathrm{Z}_{1 \mathrm{~b}}$ & $\mathrm{Z}_{2 \mathrm{a}}=\mathrm{Z}_{3 \mathrm{a}}$ & $\mathrm{Z}_{2 \mathrm{~b}}=\mathrm{Z}_{3 \mathrm{~b}}$ \\
\hline 76,63 & 56,82 & 32,37 & 13,49 \\
\hline $\mathrm{Z}_{4 \mathrm{a}}$ & $\mathrm{Z}_{4 \mathrm{~b}}$ & $\mathrm{Z}_{5}=\mathrm{Z}_{6}$ \\
\hline 54,73 & \multicolumn{2}{|c|}{13,34} & 76,27 \\
\hline
\end{tabular}

El filtro final es colocado en un soporte de 50,1 x 50,1 mm, lo que justifica la definición de sus dimensiones. La Fig. 6, muestra el filtro de 4 stubs,

FIG.6. FOTOGRAFÍA DEL FILTRO PASA BANDA DE 4 STUBS

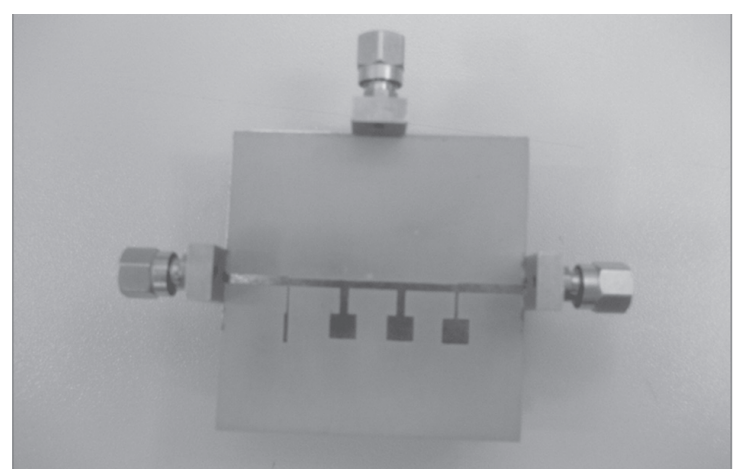




\section{RESULTADOS}

En la Fig.7, es posible ver las respuestas $\mathrm{S}_{11}$ y $\mathrm{S}_{21}$ del filtro simulado en Microwave Studio y de la medición del filtro de 4 stubs. Se puede observar que el filtro presenta una pérdida de inserción $S_{21}$ de $-3 \mathrm{~dB}$ siendo que la banda de operación es de 3,1 a $8,2 \mathrm{GHz}$. La pérdida de retorno $\mathrm{S}_{11}$ tiene un valor máximo de $-6,0 \mathrm{~dB}$ y el valor mínimo es de aproximadamente $-13 \mathrm{~dB}$ en la banda pasante. Se puede ver que las respuestas en frecuencias simuladas y prácticas tienen cierta correlación en sus características generales. El resultado obtenido en la respuesta medida del $S_{21}$ muestra un ancho de banda mayor que la respuesta simulada como en la respuesta medida. En la respuesta medida, el último cero de la frecuencia alta no está definido.

FIG.7. RESPUESTA MEDIDA Y SIMULADA DEL FILTRO DE 4. ${ }^{\circ}$ GRADO

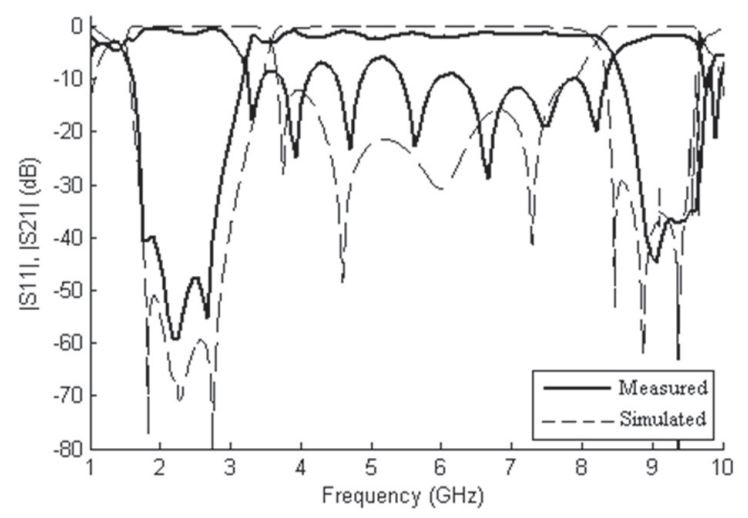

El filtro pasa banda UWB fue desarrollado para ser usado en una estructura microcinta. El sustrato tiene una constante dieléctrica de 10,5 y un grosor del dieléctrico de 0,64 mm. Las mediciones de los filtros se realizaron en un analizador de redes vectoriales modelo HP $8720 \mathrm{C}$.

\section{CONCLUSIONES}

En este estudio, el filtro pasa banda altamente selectivo ha sido propuesto para aplicaciones en el sistema UWB. Utilizando el software Microwave Studio, fue simulado el comportamiento de estos dispositivos, obteniendo los parámetros de respuestas conocidos como $S_{21}$ y $S_{11}$. Los datos de la simulación fueron comparados con las mediciones de los filtros fabricados y evaluados, se pueden considerar los resultados satisfactorios. El resultado de la simulación del filtro de 4 . grado tomó valores próximos a los proyectados en este trabajo, lo que motivó su fabricación, pero los valores medidos no fueron los esperados, debido al proceso de fabricación del impreso. En este trabajo, el método utilizado en la fabricación de la placa creo imperfecciones en los bordes y en las dimensiones más delgadas de los stubs. La banda pasante está más próxima a la proyectada, el nivel de rizado es menor, la pérdida de retorno S11 tiene un valor máximo de $-7,5 \mathrm{~dB}$ y el valor mínimo de aproximadamente $-15 \mathrm{~dB}$, y los ceros de transmisión están más próximos a los ceros deseados en el proyecto.

\section{AGRADECIMIENTOS}

Este artículo es el resultado del proyecto TaquiIla Virtual desarrollado por el grupo de investigación GIT del Instituto Tecnológico Metropolitano.

\section{REFERENCIAS}

[1] Federal Communications Commission. "Revision of part 15 of the commission's rules regarding ultra-wide transmission systems," Tech. Rep., ETDocket 98-153, FCC02-48, Apr. 2002.

[2] W.-T. Wong, Y.-S. Lin, C.-H. Wang, and C.-H Chen, "Highly Selective Microstrip Bandpass Filters for Ultra-Wideband (UWB) Applications" in IEEE Microwave Conference Proceedings, Volume 5, Issue , 4-7, Dec. 2005.

[3] Shakeri, Hosseine, "Design 3.4 GHz Wideband Bandpass Filter Using Two Section of Open Stub Connected in Shunt with Microstrip Lines." Wireless And Optical Communications Networks (WOCN), 2010 Seventh International Conference On.

[4] He, Dongi. " A compact UWB bandpass filter with ultra narrow notched band and competitive attenuation slope. Microwave Symposium Digest (MTT), 2010 IEEE MTT-S International . Page(s): 221 - 224

[5] L. Zhu; S. Sun; and W. Menzel, "Ultra-wideband (UWB) bandpass filters using multiple-mode resonator", Microwave and Wireless Components Letters, IEEE Volume 15, Issue 11, Nov. 2005 Page(s): 796 - 798

[6] C.-L. Hsu, F.-C Hsu, and J.-T. Kuo, "Microstrip bandpass filters for ultra-wideband (UWB) wireless 
communications," in IEEE MTT-S Int. Microwave Symp. Dig.CDROM, 2005.

[7] Ching, W.- T. y Ming, G.-C, “A microstrip Ultra Wideband bandpass filter with cascaded broadband bandpass and bandstop filters," IEEE Transactions on microwave theory and techniques, volume 55, No. 11, 2007.

[8] P. Cai; Z. Ma; X. Guan; X. Yang; Y. Kobayashi, T. Anada, and G. Hagiwara, "A compact UWB bandpass filter using two-section open-circuited stubs to realize transmission zeros",Microwave Conference Proceedings, Volume 5, Issue , 4-7 Dec. 2005.

[9] Tsai, L.-C., y Hsue, C.-W. (2004). Dual-band bandpass filters using equal-length coupledserial-shunted lines and z-transform technique. IEEE Trans. Microw. Theory Tech., vol.52, no.4, pp.1111-1117.

[10] CST Microwave Studio Software, versão 5.0.1, Março 19-2004.

[11] J. S. Hong and M. J. Lancaster, Microstrip Filters for RF/Microwave, Wiley, New York, 2001. 